\title{
Development of a model for physical fitness and its correlates in children and adolescents from the canton de Vaud: the PACE model
}

\section{EDUCATION / PHYSICAL EDUCATION / SPORTS AND YOUTH / SPORTS SCIENCE}

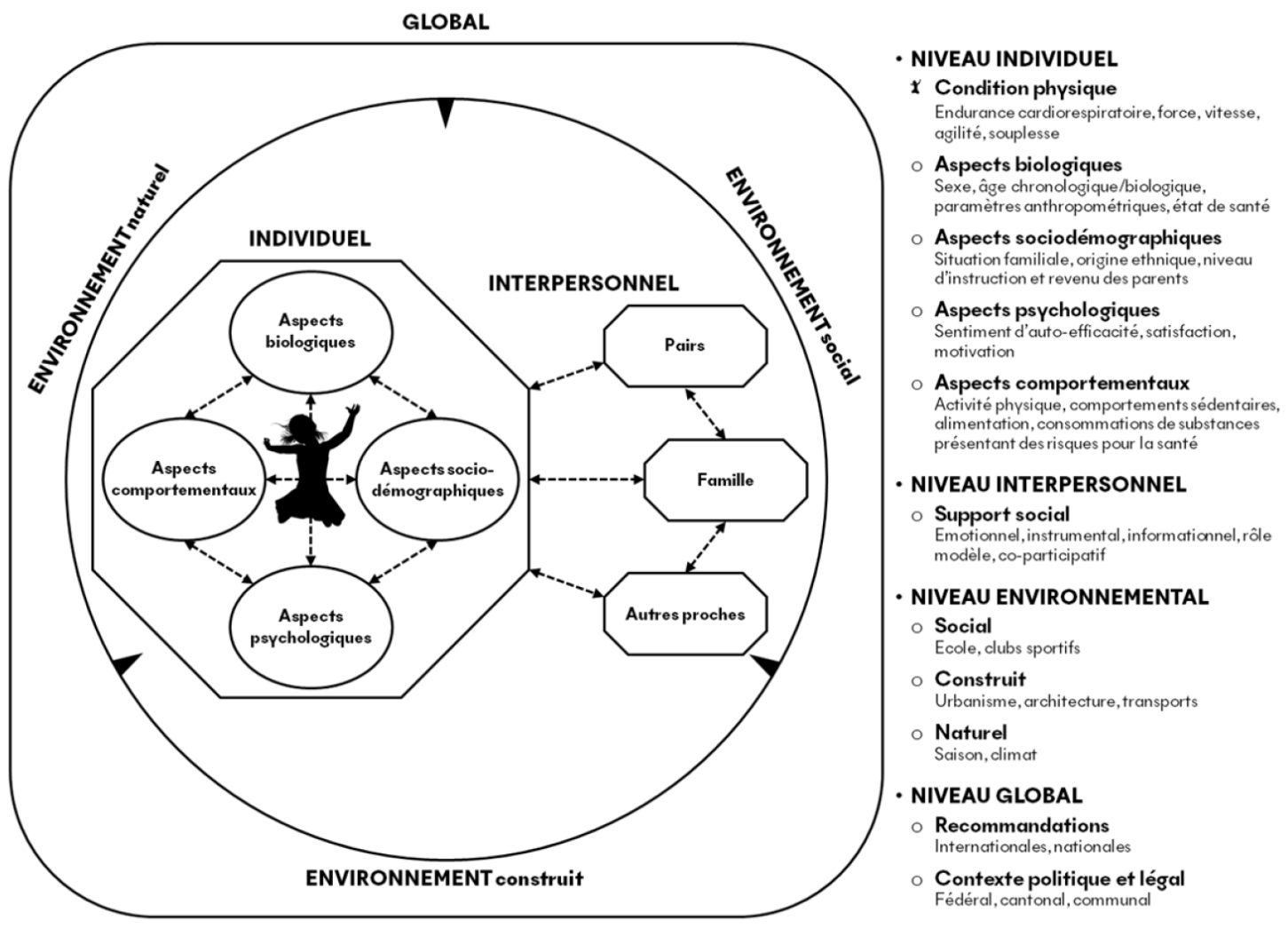

Développement d'un modèle de la condition physique et de ses corrélats chez les enfants et adolescents vaudois: le modèle pace

Guex Kenny ${ }^{1}$, Serain Emilie ${ }^{2}$, Etter Florian $^{3}$, Bize Raphaël ${ }^{4}$

${ }^{1}$ Haute Ecole de Santé Vaud (HESAV), filière physiothérapie, HES-SO Haute Ecole Spécialisée de Suisse occidentale, Lausanne, Suisse

${ }^{2}$ Haute Ecole de Santé Vaud (HESAV), unité de recherche en Santé, HES-SO Haute Ecole Spécialisée de Suisse occidentale, Lausanne, Suisse 
${ }^{3}$ Département de l'économie, de l'innovation et du sport (DEIS), Service de l'éducation physique et du sport (SEPS), Lausanne, Suisse

${ }^{4}$ Unisanté, département épidémiologie et systèmes de santé, Lausanne, Suisse

\section{Abstract}

The Lausanne Youth Olympic Games in January 2020 represent a unique opportunity for the canton of Vaud to strengthen its policy of health promotion through physical activity and sport among young people. Several factors influence physical activity and physical fitness of a population. Ecological models describe correlates of physical activity in general population and youth. However, no model describes correlates of physical fitness. To close this gap, the model of physical fitness and its correlates among children and adolescents of the canton of Vaud (PACE model) was developed. The model is organized in the following levels: global (guidelines, policy and legal context), environmental (social, built and natural environment), interpersonal (family and relatives social support) and individual (biological, sociodemographic, psychological and behavioral aspects). Perspectives on the application of the PACE model are also presented.

\section{Resumé}

Les Jeux Olympiques de la Jeunesse qui se dérouleront à Lausanne en Janvier 2020 représentent une opportunité unique pour le canton de Vaud de renforcer sa politique de promotion de la santé par l'activité physique et le sport auprès des jeunes. De multiples facteurs peuvent influencer l'activité physique et la condition physique d'une population. Différents modèles écologiques décrivent, pour la population générale et les jeunes, les corrélats de l'activité physique, mais aucun modèle ne présente ceux de la condition physique en toutes letttres. Pour combler cette lacune, nous avons développé le modèle de la $\mathrm{CP}$ et de ses corrélats chez les enfants et adolescents vaudois (modèle PACE). Le modèle est structuré selon les niveaux suivants: global (recommandations, contexte politique et légal), environnemental (environnement social, construit, naturel), interpersonnel (support social de la famille et des proches) et individuel (aspects biologiques, sociodémographiques, psychologiques, comportementaux). Des perspectives d’application du modèle PACE sont également présentées.

La forme masculine est utilisée dans cet article par souci de concision. Elle doit être comprise comme englobant l'ensemble des réalités liées au genre.

\section{Introduction}

L’organisation de grands événements sportifs a un impact positif sur l'activité physique (AP) des populations locales [1]. Les Jeux Olympiques de la Jeunesse (JOJ) 2020 de Lausanne représentent donc une opportunité unique pour le canton de Vaud de renforcer sa politique de promotion de la santé par l'activité physique et le sport auprès des jeunes. Le programme de législature 2017-2022 du Conseil d'Etat vaudois prévoit d'ailleurs d'assurer le succès des JOJ en laissant un héritage sportif et éducatif durable auprès des jeunes athlètes d'élite, ainsi qu'auprès des jeunes suisses et vaudois. 
De multiples facteurs influencent l'AP et la condition physique (CP) d'une population. Afin d'identifier les liens entre ces facteurs et le niveau d'AP, des modèles théoriques écologiques sont fréquemment utilisés [2-6]. Cependant, aucun ne présente les liens entre la CP et ses corrélats en dehors de quelques aspects individuels [7,8]. L'objectif de cet article est donc de proposer, sur la base d'une revue ciblée de la littérature scientifique, un modèle écologique de la CP et de ses corrélats chez les enfants et adolescents dans le contexte vaudois: le modèle PACE. Des perspectives concrètes d'application sont également présentées.

\section{L'activité physique et ses correlats}

L’AP correspond à la réalisation d'activités motrices volontaires entrainant une augmentation de la dépense énergétique. Chez les jeunes, l’inactivité physique est associée à l'obésité et aux risques cardiovasculaires [9-11].

La présentation des corrélats de l'AP est structurée en différents niveaux issus des modèles pour la population générale [3,4] et les jeunes [5,6]: global (recommandations, contexte politique et légal), environnemental (environnement social, construit, naturel), interpersonnel (support social de la famille et des proches) et individuel (aspects biologiques, sociodémographiques, psychologiques, comportementaux).

\section{Niveau global}

Les recommandations de l'Organisation Mondiale de la Santé (OMS) préconisent que les jeunes de 5 à 17 ans réalisent

$\geq 60 \mathrm{~min} /$ jour d'AP d'intensité modérée à soutenue [12]. En Suisse, respectivement $68 \%, 60 \%$ et 55\% des jeunes Suisse alémaniques, romands et tessinois suivent ces recommandations [13]. Aux recommandations de l'OMS, la Suisse ajoute qu'à l'âge du primaire les enfants devraient bouger davantage [14]. Au niveau de l'éducation physique et sportive en milieu scolaire (EPS), $150 \mathrm{~min} / \mathrm{sem}$ sont recommandées au primaire et $225 \mathrm{~min} / \mathrm{sem}$ au secondaire, dont 50\% devraient être réalisées à une intensité modérée à soutenue [15]. Le contexte politique et légal est un corrélat de l'AP [3]. En Suisse, la Confédération fixe, après consultation des cantons, le nombre minimal de périodes d'EPS/sem [16]. La loi vaudoise sur l'EPS stipule que les élèves de l'école obligatoire doivent avoir trois périodes/sem (135 min) et que les élèves du niveau postobligatoire devraient, en général, avoir trois périodes/sem en moyenne [17]. A noter qu'au niveau national, les questions liées au sport dépendent du Département fédéral de la défense, de la protection de la population et des sports alors que celles liées à la santé dépendent du Département fédéral de l'intérieur. Au niveau vaudois également les questions liées au sport et à la santé sont traitées par deux départements distincts: celui de l'économie, de l'innovation et du sport et celui de la santé et de l'action sociale.

\section{Niveau environnemental}

Chez les jeunes, l'environnement social comprend principalement les éléments liés à l'école et aux clubs sportifs. De par le temps passé assis, l'école est un haut lieu de sédentarité. Mais elle est surtout un cadre de choix pour engager les jeunes à réaliser suffisamment d'AP et promouvoir des comportements limitant le risque de surpoids [18-22]. Pour beaucoup de jeunes, elle est même l'occasion principale de participer à des AP au travers de l'EPS [23], qui est jugée, en Suisse, de bonne qualité, intéressante et instructive [24]. En revanche, elle ne permet pas à elle seule de respecter les recommandations, entre autres à cause du 
temps trop réduit passé à intensité modérée à soutenue [25-27]. Des interventions ciblées durant les leçons permettent d'augmenter ce temps (22) et ont un impact positif sur la CP et la santé [20,28,29]. En Suisse, un programme d'un an visant à augmenter le volume et l'intensité des AP dans le cadre scolaire (ajout de deux périodes d'EPS/sem, de courtes pauses composées de tâches motrices et de devoirs d'AP à domicile) a montré, chez des enfants de 6 à 13 ans, des effets bénéfiques sur la composition corporelle, les aptitudes aérobies et les risques cardiovasculaires [30]. Une fois l'intervention arrêtée, ces améliorations n'étaient pas maintenues sur le long terme [31]. Une intervention continue est donc nécessaire, ce qui implique des changements au niveau global.

En complément des leçons obligatoires d'EPS, 40\% des écoles suisses offrent des cours facultatifs de sport, auxquels $26 \%$ des jeunes participent [13,24]. Ces cours sont accessibles à tous les élèves, y compris les moins actifs, quel que soit leur origine ou leur milieu socioéconomique [13]. Enfin, les clubs sportifs jouent un rôle important dans la pratique de l'AP en Suisse: deux tiers des 6-11 ans, $62 \%$ des 10-14 ans et 43\% des 15-19 ans sont membres d'un club [13,24]. Dans le canton de Vaud, les jeunes de $\leq 20$ ans représentaient la moitié des membres des clubs sportifs en 2016: 10\% de plus qu'en 1996 [32].

L'environnement construit comprend les éléments liés à l'urbanisme, à l'architecture et aux transports. L’aménagement du territoire, l'accessibilité et la proximité des installations de loisir, la densité résidentielle, le potentiel piétonnier et la vitesse/densité du trafic influencent l'AP des enfants, alors que seuls l'aménagement du territoire et à la densité résidentielle influencent l'AP des adolescents [33]. Au niveau des transports, les déplacements actifs pour aller à l'école sont favorisés par des passages piétons ou des trottoirs à proximité des établissements scolaires [34]. En Suisse, les jeunes vivant en ville sont moins sportifs que ceux des communes d'agglomération ou des régions rurales [13].

L'environnement naturel comprend les aspects saisonniers et climatiques. Chez les jeunes, une baisse de l'AP et une augmentation de la sédentarité sont retrouvées en automne et en hiver [35-37]. En lien avec l'augmentation de l'AP totale et d'intensité modérée à soutenue observée au printemps, l'endurance cardiorespiratoire est plus élevée au printemps qu'en automne [35]. En Suisse, l'AP des jeunes est également plus basse en hiver [13]. Au niveau du climat, la température, les précipitations, le vent et la visibilité influencent la pratique d'AP des jeunes [38].

\section{Niveau interpersonnel}

Le support social décrit les ressources de type émotionnel, instrumental, informationnel, «rôle modèle» et co-participatif issues des interactions avec des personnes pouvant influencer le comportement de l'individu [39-43]. De manière générale, le support familial est un corrélat de l'AP chez les jeunes [3]: le rôle modèle des parents et le fait de pratiquer des AP avec eux jouent un rôle important dans le développement et la pratique de l'AP de l'enfant [40,41]. A l'adolescence, le rôle des pairs prend par ailleurs de l'importance. Les ressources émotionnelles et instrumentales sont corrélées à l'AP quel que soit l'âge [41]. En Suisse, les jeunes de 10 à 19 ans dont les parents sont sportifs font plus souvent du sport que ceux ayant des parents inactifs [24]. La proportion des jeunes de 6 à 16 ans respectant les recommandations est également plus grande chez les jeunes ayant des parents sportifs [13]. Dans le canton de Vaud, les adolescents dont les parents ne pratiquent pas d'AP sont plus nombreux à être peu actifs [7].

\section{Niveau individuel}

Le sexe, l'âge chronologique et biologique, les paramètres anthropométriques et l'état de santé font parties 
des aspects biologiques. Le sexe et l'âge sont des corrélats de l'AP [3,44]. En Suisse, les garçons sont plus actifs que les filles [24,45]. A l'adolescence, une baisse d'activité associée à une augmentation du temps passé assis est observée chez les deux sexes. Dans le canton de Vaud, une diminution de l'AP a été observée à partir de 15 ans principalement chez les filles [7]. Elle s'explique par une diminution des cours d'EPS et des pratiques sportives. Au niveau des paramètres anthropométriques, aucun lien n'a été trouvé avec l'AP [3]. Concernant l'état de santé, les jeunes vaudois faisant partie d'un club et faisant du sport tous les jours s'estiment en meilleure santé physique [7].

Parmi les aspects sociodémographiques, on retrouve la situation familiale (parents mariés ou séparés), l'origine ethnique, le niveau d'instruction et de revenu des parents. La situation familiale n'est pas identifiée comme un corrélat de l'AP [3], alors que le fait d'être caucasien oui [46,47]. En Suisse, les jeunes issus de la migration, ceux dont le niveau de formation des parents ne dépasse pas l'école obligatoire, et ceux dont le revenu du ménage est bas sont moins actifs [24,45].

Le sentiment d'auto-efficacité, la satisfaction et la motivation font parties des aspects psychologiques. L'auto-efficacité et la satisfaction à faire des AP contribuent à soutenir l'AP chez les jeunes [48-52]. Chez les adolescents, un effet synergique et compensatoire entre l'auto-efficacité et le support social des pairs a été démontré: un faible niveau d'auto-efficacité peut être compensé par le support des amis pour développer l'intention de réaliser des AP [48]. A l'inverse, un manque de support des pairs n'est pas un désavantage si le sentiment d'auto-efficacité est élevé. En Suisse, nous disposons de données sur la motivation des jeunes à faire du sport: le plaisir ressort comme raison principale, suivi du fait d'être en bonne forme physique et bien entraîné et de partager des moments de convivialité [24]. Au niveau scolaire en revanche, un déclin de la motivation à l'égard de l'EPS a récemment été montré chez des jeunes suisses de 8 à 12 ans (53). De plus, la motivation autonome de ces jeunes serait en lien avec leur comportement en matière d'AP. Parmi les aspects comportementaux, on retrouve les comportements sédentaires, l'alimentation et les consommations de substances présentant des risques pour la santé. La sédentarité peut être mesurée indirectement en estimant le temps passé devant les écrans [7,54]. En 2014, les jeunes suisses de 11 à 15 ans passaient $4.4 \mathrm{~h} /$ jour de semaine devant un écran et $7.4 \mathrm{~h} /$ jour de week-end [55], alors que le fait de passer respectivement $\geq 4.5 \mathrm{~h} /$ jour et $\geq 7.5 \mathrm{~h} /$ jour devant un écran est associé à une pratique moindre d'activités physiques. A la fin des années 90, les jeunes vaudois passaient $<2 \mathrm{~h} /$ jour devant la télévision avec une tendance à la baisse dès 16 ans, en lien avec une modification du type de loisirs [7]. De nos jours, le caractère mobile des écrans rend moins fiable cette méthode d'estimation de la sédentarité [54].

Au niveau des comportements alimentaires, seul 10\% des jeunes suisses de 11 à 15 ans mangent des fruits et légumes plusieurs fois/jour [54], alors que le fait d'en manger moins d'une fois/jour est associé avec une probabilité accrue d'être inactif. Au niveau des consommations de substances à risque, les jeunes suisses qui fument du tabac au moins une fois/ semaine ont plus de chance d'être inactifs. En revanche, aucune association n’a été montrée avec la consommation d'alcool [54].

\section{La condition physique}

La CP correspond à la capacité de réaliser des AP et fait référence à différentes qualités: l'endurance, la force, la vitesse, l'agilité et la souplesse [56]. De faibles aptitudes cardiorespiratoires et musculosquelettiques à l'adolescence sont associées à un risque augmenté de mort prématurée [57,58]. A l'inverse, ces aptitudes sont liées à une diminution du risque d'obésité et de maladies cardiométaboliques, 
ainsi qu'à une bonne santé osseuse et mentale [56,59-61]. La CP représente donc un important indicateur de santé. De plus, elle est positivement associée aux performances scolaires [62-64].

\section{Evaluation de la condition physique}

La batterie de tests Eurofit est considérée comme la méthode de choix pour évaluer la CP chez les jeunes [65-67]. Elle évalue l'équilibre, la souplesse, la vitesse et l'agilité de course, la vitesse du haut du corps, l'endurance musculaire du tronc, la puissance musculaire des membres inférieurs, l'endurance cardiorespiratoire, la force des membres supérieurs et l'endurance musculaire des membres supérieurs. Elle est peu coûteuse, fiable, valide, simple à administrer et demande peu de matériel et de personnel. Sur la base des normes européennes [67], le niveau de chaque jeune de 9 à 17 ans peut être catégorisé: <20ème centile: très bas/faible; 20-40ème centile: bas/faible; 40-60ème centile: modéré; 60-80ème centile: élevé/bon; > 80ème centile: très élevé/bon. Le quintile le plus bas représente un seuil au-dessous duquel le jeune est défini comme ayant un risque disproportionné de maladies futures [68].

\section{Etude vaudoise}

Entre 1996 et 1997, une étude a mesuré la CP d'élèves vaudois de 9 à 19 ans à l'aide de la batterie Eurofit et a étudié les relations entre cette dernière et des aspects biologiques (sexe, âge, paramètres anthropométriques) et comportementaux (AP) [7,8,69,70]. Par rapport aux normes européennes, les niveaux obtenus se situent entre les percentiles 30 et 95 pour les filles, et 40 et 95 pour les garçons (Figure 1) [67].

Deux autres études ont testé certains paramètres de la $C P$ chez des jeunes suisses [30,71]. La première a mesuré l'endurance cardiorespiratoire d'un groupe mixte de 9 et 11 ans [30]. La seconde a évalué quatre paramètres de la batterie Eurofit chez des jeunes de 11 ans [71]. Leurs résultats sont comparables à ceux de l'étude vaudoise. 

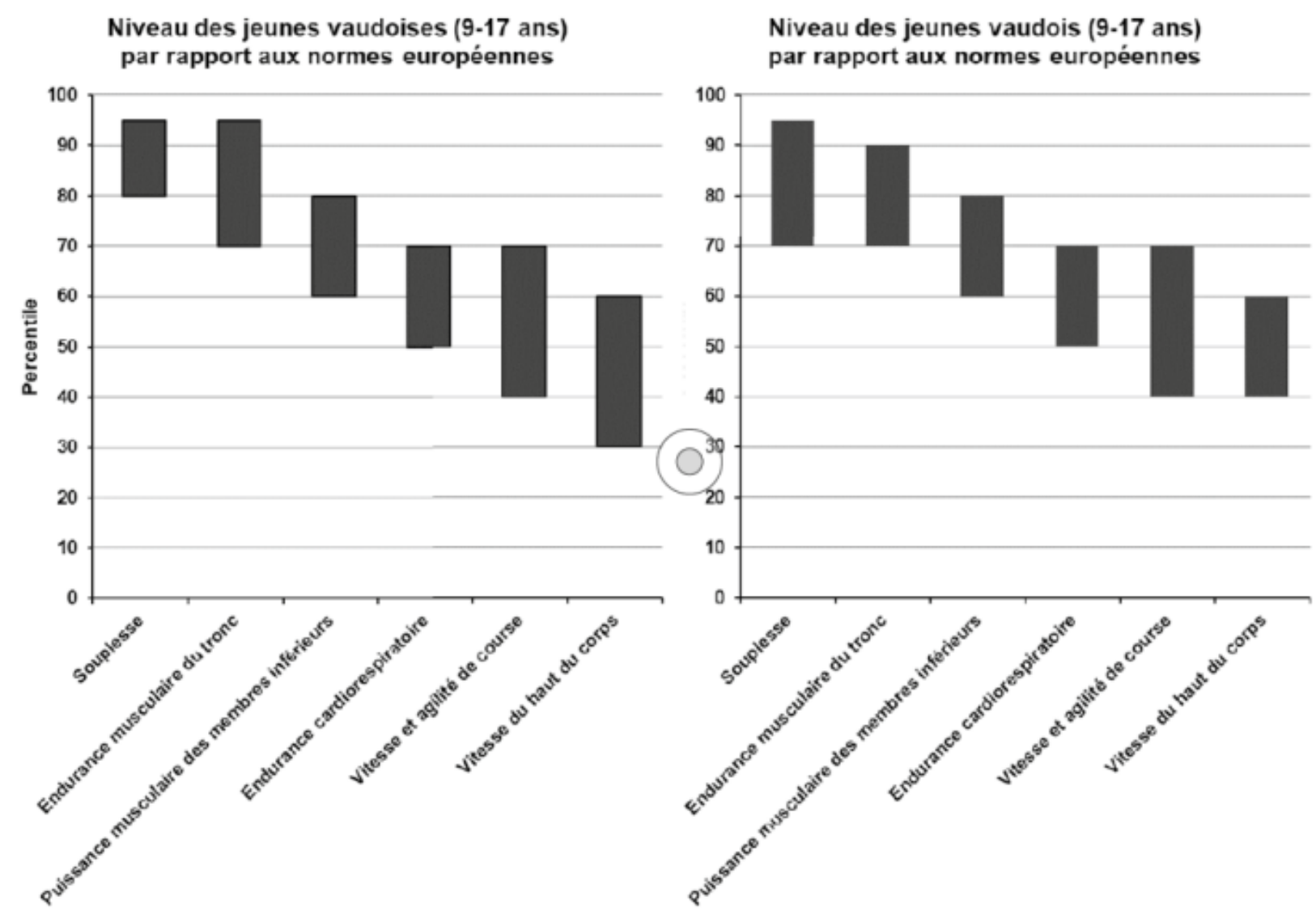

Figure 1: Niveau des jeunes vaudois par rapport aux normes européennes. 


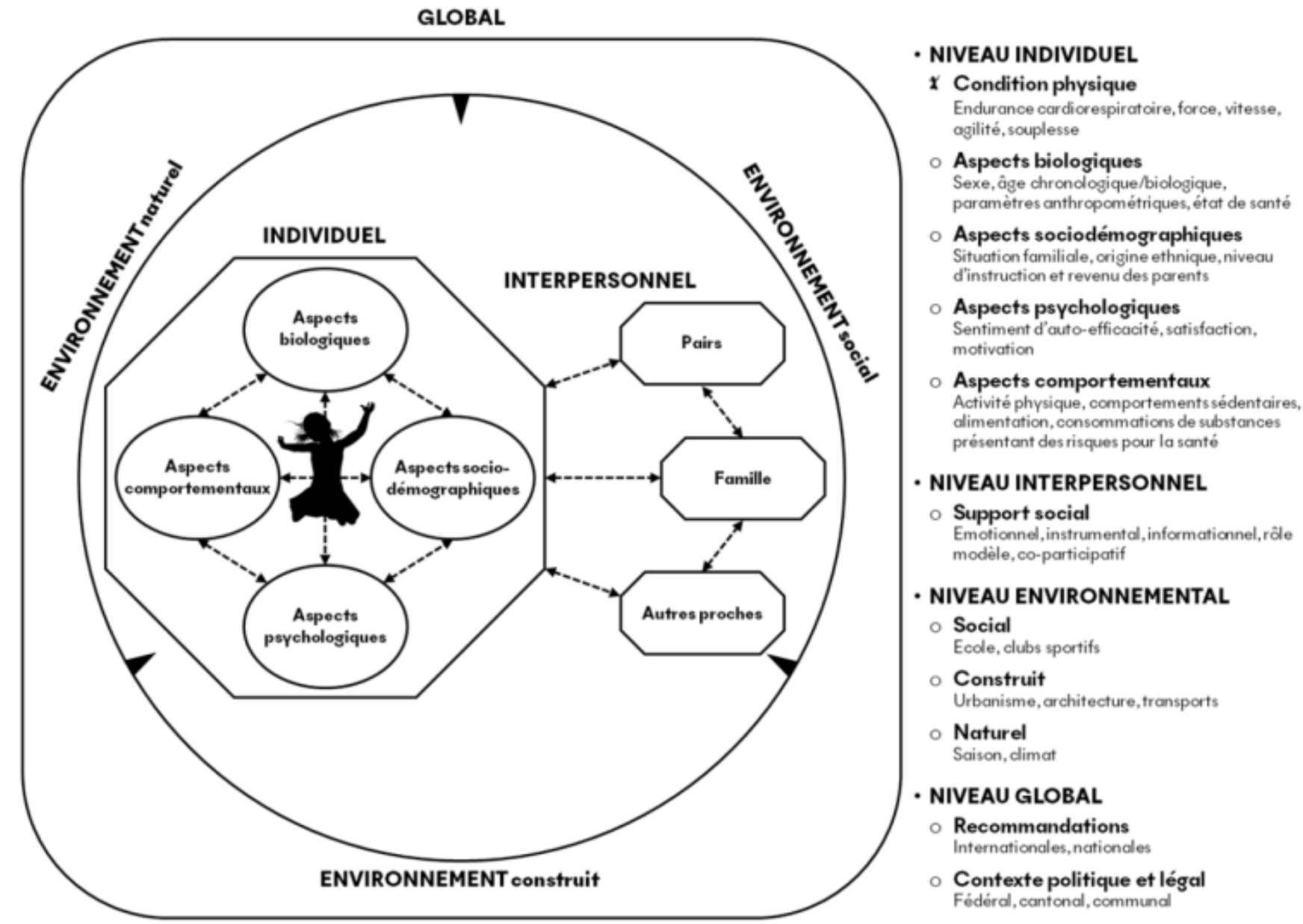

Figure 2: Modèle PACE.

Evolution de la condition physique

Pour certains auteurs, la génération actuelle de jeunes souffrirait d'un «analphabétisme physique» [72]. En effet, les résultats des jeunes de plusieurs pays montrent un déclin de certaines qualités de la CP sur des périodes allant de cinq à 26 ans [73-75]. De plus, une diminution de l'endurance cardiorespiratoire de près de 0.5\%/an a été observée entre 1980 et 2000 [76]. En Suisse, il n'existe pas de données sur l'évolution de la CP des jeunes. Cependant, les résultats de l'étude vaudoise de 1996-1997 pourraient servir de base pour l'évaluer [7].

\section{Corrélats de la condition physique}

Il n'existe aucun modèle présentant les liens entre la $\mathrm{CP}$ et ses corrélats en dehors de certains aspects individuels présentés dans l'étude vaudoise [7,8].

Les résultats de cette dernière montrent que les filles sont plus performantes aux tests d'agilité et de souplesse et les garçons aux tests d'endurance, de vitesse et de puissance [7]. L'évolution des performances selon l'âge chronologique est comparable chez les deux sexes jusqu'à la puberté, durant laquelle les performances ont tendance à se stabiliser chez les filles et continuer à augmenter chez les garçons. Ces observations sont en lien avec celles issues des normes européennes [67]. Au niveau des paramètres 
anthropométriques, la taille est positivement corrélée à plusieurs qualités de la CP chez les filles (vitesse et agilité de course, vitesse du haut du corps, puissance musculaire des membres inférieurs) et chez les garçons (vitesse et agilité de course, vitesse du haut du corps, puissance musculaire des membres inférieurs, endurance musculaire du tronc, endurance cardiorespiratoire), alors que le poids est positivement corrélé à certaines qualités (vitesse et agilité de course, endurance musculaire du tronc, puissance musculaire des membres inférieurs) uniquement chez les garçons.

L'étude vaudoise n'a trouvé aucune relation entre la CP et l'AP rapportée par les jeunes [7]. D’autres études ont trouvé un lien faible à modéré [19,77]. L’AP journalière n'expliquerait, par exemple, qu'une faible partie des capacités aérobies [21,78]. Malgré cela, les enfants les moins actifs augmenteraient de manière notable leur capacité aérobie en augmentant leur AP [20,21,79]. De plus, la tendance générale à une diminution de CP serait en partie explicable par le fait que l'AP réalisée dans le cadre de l'EPS, des déplacements actifs et du sport organisé, s’affaiblit dans de nombreux pays, alors que les jeunes manifestent un désir fort et constant d'être actifs [80].

\section{Le modèle pace}

Ces résultats montrent qu'il est crucial d'identifier les corrélats de la CP non seulement au niveau individuel, mais également à tous les niveaux du modèle écologique. Or, la littérature scientifique présente soit des modèles écologiques décrivant l'AP et ses corrélats, soit un modèle décrivant certains aspects individuels influençant la $\mathrm{CP}$. Pour combler cette lacune, nous avons développé le modèle de la $\mathrm{CP}$ et de ses corrélats chez les enfants et adolescents vaudois (modèle PACE) (Figure 2) [81]. Il est adapté des modèles connus de l'AP et de la CP [3-6,8]. Il est écologique car il inclut les relations entre le jeune, ses caractéristiques individuelles, ses relations avec ses proches, ainsi que l'influence de son environnement et du contexte dans lequel il évolue.

\section{Perspectives}

Près d'un quart de siècle après les premières valeurs récoltées, des mesures de la $\mathrm{CP}$ des élèves vaudois pourraient être mises en place dans le but de mesurer le niveau actuel des jeunes, de le comparer au niveau passé et de comparer l'évolution de la CP aux évolutions observées ailleurs en Europe. De plus, la mise en place d'un questionnaire basé sur le modèle PACE permettrait de vérifier l'existence de corrélats de la CP à tous les niveaux du modèle écologique, et d'ainsi apporter une validation supplémentaire au modèle PACE. Les résultats pourraient permettre de proposer et d'évaluer différentes stratégies préventives à tous les niveaux du modèle écologique (p. ex. via l'urbanisme, l'accès aux clubs sportifs, les recommandations en matière d'AP, etc.) visant à stimuler et encourager les jeunes dont la CP les expose à des risques accrus de maladie. L'organisation des JOJ peut servir de tremplin à de tels projets et représente une chance unique de faire du sport de performance un vecteur de la promotion de l'activité physique et du sport pour tous et pour la santé.

\section{Corresponding authorם}


Kenny Guex

School of Health Sciences (HESAV),

HES-SO University of Applied Sciences

and Arts Western Switzerland

Lausanne, Switzerland

kenny.guex@hesav.ch

+41213168046

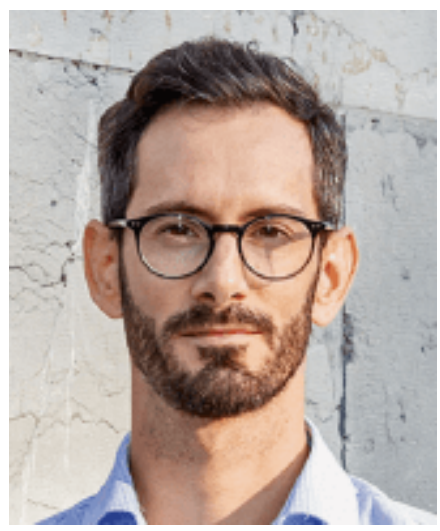

\section{References}

1. Murphy NM, Bauman A. Mass sporting and physical activity events-are they "bread and circuses" or public health interventions to increase population levels of physical activity? Journal of physical activity \& health. 2007;4(2):193-202.

2. Bronfenbrenner U. The ecology of human development : experiments by nature and design. Cambridge, Mass.; London: Harvard University Press; 1979.xvii,330p p.

3. Bauman AE, Reis RS, Sallis JF, Wells JC, Loos RJ, Martin BW, et al. Correlates of physical activity: why are some people physically active and others not? Lancet. 2012;380(9838):258-71.

4. Sallis JF, Cervero RB, Ascher W, Henderson KA, Kraft MK, Kerr J. An ecological approach to creating active living communities. Annu Rev Public Health. 2006;27:297-322.

5. Mehtala MA, Saakslahti AK, Inkinen ME, Poskiparta ME. A socio-ecological approach to physical activity interventions in childcare: a systematic review. Int J Behav Nutr Phys Act. 2014;11:22.

6. O'Connor J, Alfrey L, Payne P. Beyond games and sports: a socio-ecological approach to physical education. Sport Educ Soc. 2012;17(3):365-80.

7. Narring F, Berthoud A, Cauderay M, Favre M, Michaud P-A. Condition physique et pratiques sportives des jeunes dans le canton de Vaud. Lausanne: Institut universitaire de médecine sociale et préventive; 1997.

8. Michaud PA, Narring F, Cauderay M, Cavadini C. Sports activity, physical activity and fitness of 9- to 19-year-old teenagers in the canton of Vaud (Switzerland). Schweiz Med Wochenschr. 1999;129(18):691-9.

9. Andersen LB, Harro M, Sardinha LB, Froberg K, Ekelund U, Brage S, et al. Physical activity and clustered cardiovascular risk in children: a cross-sectional study (The European Youth Heart Study). Lancet. 2006;368(9532):299-304.

10. Ekelund U, Luan J, Sherar LB, Esliger DW, Griew P, Cooper A, et al. Moderate to vigorous physical activity and sedentary time and cardiometabolic risk factors in children and adolescents. JAMA. 2012; 307(7):704-12.

11. Froberg K, Andersen LB. Mini review: physical activity and fitness and its relations to cardiovascular disease risk factors in children. Int J Obes (Lond). 2005;29 Suppl 2:S34-9.

12. WHO. Global recommendations on physical activity for health. Geneva, Switzerland: World Health Organization; 2010. p. 23-7.

13. Bringolf-Isler B, Probst-Hensch N, Kayser B, Suggs S. Schlussbericht zur SOPHYA-Studie. Basel: Swiss Tropical and Public Health Institute; 2016.

14. OFSPO. Activité physique et santé des enfants et des adolescents: Recommandations pour la Suisse. Macolin, Suisse; 2013. 
15. Hills AP, Dengel DR, Lubans DR. Supporting public health priorities: recommendations for physical education and physical activity promotion in schools. Prog Cardiovasc Dis. 2015;57(4):368-74.

16. Loi sur l'encouragement du sport, LESp, RS 415.0 (2011).

17. Loi sur l'éducation physique et le sport, 415.01 (2012).

18. Pate RR, Davis MG, Robinson TN, Stone EJ, McKenzie TL, Young JC, et al. Promoting physical activity in children and youth: a leadership role for schools: a scientific statement from the American Heart Association Council on Nutrition, Physical Activity, and Metabolism (Physical Activity Committee) in collaboration with the Councils on Cardiovascular Disease in the Young and Cardiovascular Nursing. Circulation. 2006;114(11):1214-24.

19. Jaakkola T, Huhtiniemi M, Salin K, Seppala S, Lahti J, Hakonen H, et al. Motor competence, perceived physical competence, physical fitness, and physical activity within Finnish children. Scand J Med Sci Sports. 2019.

20. Seljebotn PH, Skage I, Riskedal A, Olsen M, Kvalo SE, Dyrstad SM. Physically active academic lessons and effect on physical activity and aerobic fitness. The Active School study: A cluster randomized controlled trial. Prev Med Rep. 2019;13:183-8.

21. Kristensen PL, Moeller NC, Korsholm L, Kolle E, Wedderkopp N, Froberg K, et al. The association between aerobic fitness and physical activity in children and adolescents: the European youth heart study. Eur J Appl Physiol. 2010;110(2):267-75.

22. Lonsdale C, Rosenkranz RR, Peralta LR, Bennie A, Fahey P, Lubans DR. A systematic review and metaanalysis of interventions designed to increase moderate-to-vigorous physical activity in school physical education lessons. Prev Med. 2013;56(2):152-61.

23. Meyer U, Roth R, Zahner L, Gerber M, Puder JJ, Hebestreit H, et al. Contribution of physical education to overall physical activity. Scand J Med Sci Sports. 2013;23(5):600-6.

24. Lamprecht Markus AF, Doris Wiegand, Hanspeter Stamm. Sport Suisse 2014, Rapport sur les enfants et adolescents. Observatoire sport et activité physique Suisse. 2014.

25. Gubelmann C, Marques-Vidal P, Bringolf-Isler B, Suggs LS, Vollenweider P, Kayser B. Correlates of weekday compliance to physical activity recommendations in Swiss youth non-compliant in weekend days. Prev Med Rep. 2018;9:86-91.

26. Hollis JL, Sutherland R, Williams AJ, Campbell E, Nathan N, Wolfenden L, et al. A systematic review and meta-analysis of moderate-to-vigorous physical activity levels in secondary school physical education lessons. Int J Behav Nutr Phys Act. 2017;14(1):52.

27. Hollis JL, Williams AJ, Sutherland R, Campbell E, Nathan N, Wolfenden L, et al. A systematic review and meta-analysis of moderate-to-vigorous physical activity levels in elementary school physical education lessons. Prev Med. 2016;86:34-54.

28. Larsen MN, Nielsen CM, Helge EW, Madsen M, Manniche V, Hansen L, et al. Positive effects on bone mineralisation and muscular fitness after 10 months of intense school-based physical training for children aged 8-10 years: the FIT FIRST randomised controlled trial.

Br J Sports Med. 2018;52(4):254-60.

29. de Greeff JW, Hartman E, Mullender-Wijnsma MJ, Bosker RJ, Doolaard S, Visscher C. Effect of Physically Active Academic Lessons on Body Mass Index and Physical Fitness in Primary School Children. J Sch Health. 2016;86(5):346-52.

30. Kriemler S, Zahner L, Schindler C, Meyer U, Hartmann T, Hebestreit H, et al. Effect of school based physical activity programme (KISS) on fitness and adiposity in primary schoolchildren: cluster randomised controlled trial. Bmj. 2010;340:c785.

31. Meyer U, Schindler C, Zahner L, Ernst D, Hebestreit H, van Mechelen W, et al. Long-term effect of a school-based physical activity program (KISS) on fitness and adiposity in children: a clusterrandomized controlled trial. PLoS One. 2014;9(2):e87929. 
32. SEPS, STATVD. Portrait des clubs sportifs vaudois 2016. Lausanne, Suisse; 2017.

33. Ding D, Sallis JF, Kerr J, Lee S, Rosenberg DE. Neighborhood environment and physical activity among youth a review. Am J Prev Med. 2011;41(4):442-55.

34. Hume C, Timperio A, Salmon J, Carver A, Giles-Corti B, Crawford D. Walking and cycling to school: predictors of increases among children and adolescents. American journal of preventive medicine. 2009;36(3):195-200.

35. Hjorth MF, Chaput JP, Michaelsen K, Astrup A, Tetens I, Sjodin A. Seasonal variation in objectively measured physical activity, sedentary time, cardio-respiratory fitness and sleep duration among 8-11 year-old Danish children: a repeated-measures study. BMC Public Health. 2013;13:808.

36. Rich C, Griffiths LJ, Dezateux C. Seasonal variation in accelerometer-determined sedentary behaviour and physical activity in children: a review. Int J Behav Nutr Phy. 2012;9.

37. Atkin AJ, Sharp SJ, Harrison F, Brage S, Van Sluijs EMF. Seasonal Variation in Children's Physical Activity and Sedentary Time. Med Sci Sport Exer. 2016;48(3):449-56.

38. Harrison F, Goodman A, van Sluijs EMF, Andersen LB, Cardon G, Davey R, et al. Weather and children's physical activity; how and why do relationships vary between countries? Int J Behav Nutr Phys Act. 2017;14(1):74.

39. Langford CP, Bowsher J, Maloney JP, Lillis PP. Social support: a conceptual analysis. J Adv Nurs. 1997;25(1):95-100.

40. Yao CA, Rhodes RE. Parental correlates in child and adolescent physical activity: a meta-analysis. Int J Behav Nutr Phys Act. 2015;12:10.

41. Pugliese J, Tinsley B. Parental socialization of child and adolescent physical activity: a meta-analysis. J Fam Psychol. 2007;21(3):331-43.

42. Stroebe W, Stroebe M. The social psychology of social support. Social psychology: handbook of basic principles. New-York: Guilford Press; 1996.

43. Glanz K, Rimer BK, Viswanath K, Orleans CT. Health behavior and health education theory, research, and practice. San Francisco, CA: Jossey-Bass,; 2008. Available from:

http://kcl.eblib.com/patron/FullRecord.aspx?p=353367.

44. Wilk P, Clark AF, Maltby A, Smith C, Tucker P, Gilliland JA. Examining individual, interpersonal, and environmental influences on children's physical activity levels. SSM Popul Health. 2018;4:76-85.

45. Bringolf-Isler B, Probst-Hensch N., Kayser B., Suggs S. Sophya Studies. Swiss TPH, Institut tropical et de santé publique Suisse. 2016.

46. Uijtdewilligen L, Nauta J, Singh AS, van Mechelen W, Twisk JW, van der Horst K, et al. Determinants of physical activity and sedentary behaviour in young people: a review and quality synthesis of prospective studies. Br J Sports Med. 2011;45(11):896-905.

47. Craggs C, Corder K, van Sluijs EM, Griffin SJ. Determinants of change in physical activity in children and adolescents: a systematic review. Am J Prev Med. 2011;40(6):645-58.

48. Hamilton K, Warner LM, Schwarzer R. The Role of Self-Efficacy and Friend Support on Adolescent Vigorous Physical Activity. Health education \& behavior: the official publication of the Society for Public Health Education. 2017;44(1):175-81.

49. Verloigne M, Cardon G, De Craemer M, D’Haese S, De Bourdeaudhuij I. Mediating Effects of SelfEfficacy, Benefits and Barriers on the Association between Peer and Parental Factors and Physical Activity among Adolescent Girls with a Lower Educational Level. PloS one. 2016;11(6):e0157216.

50. Garcia JM, Sirard JR, Larsen R, Bruening M, Wall M, Neumark-

Sztainer D. Social and Psychological Factors Associated With Adolescent Physical Activity. Journal of physical activity \& health. 2016;13(9):957-63.

51. Kim GS, Lee CY, Kim IS, Lee TH, Cho E, Lee H, et al. Dyadic Effects of Individual and Friend on Physical Activity in College Students. Public health nursing (Boston, Mass). 2015;32(5):430-9.

52. Shen B, Centeio E, Garn A, Martin J, Kulik N, Somers C, et al. Parental social support, perceived 
competence and enjoyment in school physical activity. Journal of sport and health science. 2018;7(3):346-52.

53. Chanal J, Cheval B, Courvoisier DS, Paumier D. Developmental relations between motivation types and physical activity in elementary school children. Psychol Sport Exerc. 2019;43:233-42.

54. Archimi A, Eichenberger Y, Kretschmann A., Delgrande Jordan M, Bacher E. Habitudes alimentaires, activité physique, usage des écrans et statut pondéral chez les élèves de 11 à 15 ans en Suisse Résultats de l'enquête «Health Behaviour in School-aged Children» (HBSC) 2014 et évolution au fil du temps. Lausanne; 2016.

55. Archimi A. EY, Kretschmann A., Delgrande Jordan M Habitudes alimentaires, activité physique, usage des écrans et statu pondéral chez les élèves de 11 à 15 ans en Suisse. Résultats de l'enquête "Health Behaviour in School-aged Children” (HBSC) 2014 et évolution au fil du temps. 2014.

56. Ortega FB, Ruiz JR, Castillo MJ, Sjostrom M. Physical fitness in childhood and adolescence: a powerful marker of health. Int J Obes (Lond). 2008;32(1):1-11.

57. Ortega FB, Silventoinen K, Tynelius P, Rasmussen F. Muscular strength in male adolescents and premature death: cohort study of one million participants. Bmj. 2012;345:e7279.

58. Hogstrom G, Nordstrom A, Nordstrom P. Aerobic fitness in late adolescence and the risk of early death: a prospective cohort study of 1.3 million Swedish men. Int J Epidemiol. 2016;45(4):1159-68.

59. Ortega FB, Labayen I, Ruiz JR, Kurvinen E, Loit HM, Harro J, et al. Improvements in fitness reduce the risk of becoming overweight across puberty. Med Sci Sports Exerc. 2011;43(10):1891-7.

60. Smith JJ, Eather N, Morgan PJ, Plotnikoff RC, Faigenbaum AD, Lubans DR. The health benefits of muscular fitness for children and adolescents: a systematic review and meta-analysis. Sports Med. 2014;44(9):1209-23.

61. Ruiz JR, Castro-Pinero J, Artero EG, Ortega FB, Sjostrom M, Suni J, et al. Predictive validity of healthrelated fitness in youth: a systematic review. Br J Sports Med. 2009;43(12):909-23.

62. Archer T, Garcia D. Physical Exercise Influences Academic Performance and Well-being in Children and Adolescents. Int J Sch Cogn Psychol. 2014;1(1).

63. del Pozo FJF, Alonso JV, Alvarez MV, Orr S, Cantarero FJL. Physical fitness as an indicator of health status and its relationship to academic performance during the prepubertal period. Health Promot Perspe. 2017;7(4):197-204.

64. Donnelly JE, Hillman CH, Castelli D, Etnier JL, Lee S, Tomporowski P, et al. Physical Activity, Fitness, Cognitive Function, and Academic Achievement in Children: A Systematic Review. Med Sci Sports Exerc. 2016;48(6):1197-222.

65. Kemper HCG, VanMechelen W. Physical fitness testing of children: A European perspective. Pediatric Exercise Science. 1996;8(3):201-14.

66. Tomkinson GR, Olds TS, Borms J. Who are the Eurofittest? Med Sport Sci. 2007;50:104-28.

67. Tomkinson GR, Carver KD, Atkinson F, Daniell ND, Lewis LK, Fitzgerald JS, et al. European normative values for physical fitness in children and adolescents aged 9-17 years: results from 2779165 Eurofit performances representing 30 countries. Br J Sports Med. 2017.

68. Ortega FB, Lavie CJ, Blair SN. Obesity and Cardiovascular Disease. Circ Res. 2016;118(11):1752-70.

69. Narring F, Cauderay M, Cavadini C, Michaud PA. Physical fitness and sport activity of children and adolescents: methodological aspects of a regional survey. Soz Praventivmed. 1999;44(2):44-54.

70. Cauderay M, Narring F, Michaud PA. A cross-sectional survey assessing physical fitness of 9-to 19year-old girls and boys in Switzerland. Pediatric Exercise Science. 2000;12(4):398-412.

71. Schmid M, Romann M, Kriemler S, Zahner L. Wie kan die Fitness von Schulkindern gemessen werden? Schweizerische Zeitschrift für Sportmedizin und Sporttraumatologie. 2007;55(2):52-61.

72. Faigenbaum AD, Lloyd RS, MacDonald J, Myer GD. Citius, Altius, Fortius: beneficial effects of resistance training for young athletes: Narrative review. Br J Sports Med. 2016;50(1):3-7.

73. Moliner-Urdiales D, Ruiz JR, Ortega FB, Jimenez-Pavon D, Vicente- Rodriguez G, Rey-Lopez JP, et al. 
Secular trends in health-related physical fitness in Spanish adolescents: the AVENA and HELENA studies. J Sci Med Sport. 2010;13(6):584-8.

74. Runhaar J, Collard DC, Singh AS, Kemper HC, van Mechelen W, Chinapaw M. Motor fitness in Dutch youth: differences over a 26-year period (1980-2006). J Sci Med Sport. 2010;13(3):323-8.

75. Cohen DD, Voss C, Taylor MJ, Delextrat A, Ogunleye AA, Sandercock GR. Ten-year secular changes in muscular fitness in English children. Acta Paediatr. 2011;100(10):e175-7.

76. Tomkinson GR, Leger LA, Olds TS, Cazorla G. Secular trends in the performance of children and adolescents (1980-2000): an analysis of 55 studies of the 20m shuttle run test in 11 countries. Sports Med. 2003;33(4):285-300.

77. Martínez-Vizcaíno V, Sánchez-López M. Relationship Between Physical Activity and Physical Fitness in Children and Adolescents. Rev Esp Cardiol. 2008;61(2):108-11.

78. Dencker M, Thorsson O, Karlsson MK, Linden C, Svensson J, Wollmer P, et al. Daily physical activity and its relation to aerobic fitness in children aged 8-11 years. Eur J Appl Physiol. 2006;96(5):587-92.

79. Brage S, Wedderkopp N, Ekelund U, Franks PW, Wareham NJ, Andersen LB, et al. Features of the metabolic syndrome are associated with objectively measured physical activity and fitness in Danish children: the European Youth Heart Study (EYHS). Diabetes Care. 2004;27(9):2141-8.

80. Dollman J, Norton K, Norton L. Evidence for secular trends in children's physical activity behaviour. Br J Sports Med. 2005;39(12):

892-7; discussion 7.

81. Alligood MR, Marriner-Tomey A. Nursing theorists and their work. 7th ed. Maryland Heights, Mo.: Mosby/Elsevier; 2010. xviii, 797 p. p. 